\title{
A Rare Case of Posterior Horseshoe Abscess Extending to Anterolateral Extraperitoneal Compartment: Anatomical and Technical Considerations
}

\author{
Christianna Oikonomou ${ }^{1}$, Periklis Alepas ${ }^{2}$, Stelios Gavriil ${ }^{2}$, Dimitrios Kalliouris ${ }^{3}$, Konstantinos Manesis ${ }^{3}$, \\ Petros Bouboulis ${ }^{3}$, Dimitrios Filippou ${ }^{1}$, Panagiotis Skandalakis ${ }^{1}$ \\ ${ }^{1}$ National Kapodistrian University Athens, Athens; ${ }^{2} 5$ th Surgery Clinic IASO General, Athens; ${ }^{3} 2$ nd Department of Surgery, 417 NIMTS \\ Hospital, Athens, Greece
}

Perianal abscess and fistula are 2 distinct entities that share a common pathology. A horseshoe fistulous abscess, a complex type of these conditions, occurs when the suppurative inflammation spreads through the deep anal space to the bilateral ischiorectal fossae. Following the intersphincteric plane, this infection may extend to the pararectal space, forming a supralevator abscess. We present a very rare case involving a 52-year-old male patient who was admitted to our surgical department with an extraperitoneal purulent inflammation as a complication following multiple drainage procedures for a posterior horseshoe abscess. Emphasis is given to the anatomical and technical considerations of eradication of anorectal sepsis and the management of complex fistula-in-ano along with a concise review of the literature.

Keywords: Horseshoe abscess; Supralevator; Extraperitoneal space; Transsphincteric fistula

\section{INTRODUCTION}

Anorectal abscesses and fistulas are potentially debilitating but rarely life-threatening conditions. They usually originate from an infection in the cryptoglandular epithelium and, based on their location, are classified into perianal, ischiorectal, intersphincteric and supralevator [1]. According to the literature, perianal and ischiorectal abscesses are the most common [2]. A horseshoe abscess is caused by the lateral spread of an ischioanal abscess through the conjoint longitudinal muscle in the anterior or posterior midline, or the deep anterior or posterior anal space. Cephalad extension of the suppurative process in the supralevator space and extraperitoneal compartments is a rare complication. The

Received: January 27, 2018 - Accepted: March 5, 2018

Correspondence to: Christianna Oikonomou, M.D.

National Kapodistrian University Athens, 75, Mikras Asias St. Goudi, Athens 11527, Greece

Tel: +306948447999

E-mail: christianna.oikonomou@gmail.com

ORCID code: https://orcid.org/0000-0001-5773-5276

(C) 2019 The Korean Society of Coloproctology

This is an open-access article distributed under the terms of the Creative Commons Attribution NonCommercial License (http://creativecommons.org/licenses/by-nc/4.0) which permits unrestricted noncommercial use, distribution, and reproduction in any medium, provided the original work is properly cited. complex anatomic planes and the occult nature of the infection require a thorough knowledge of the relevant anatomy and a high index of suspicion regarding the diagnosis and management.

\section{CASE REPORT}

An otherwise healthy 52-year-old male patient was admitted to our surgical department from a public hospital with sepsis following multiple drainage procedures of a perianal abscess. He presented with fever, cramping lower abdominal pain and severe pain around the area of the buttocks. The physical examination revealed tenderness upon deep palpation of the right lower abdominal quadrant with no evidence of peritoneal irritation. An extensive perianal surgical wound was revealed, indicating the previous drainage procedures were performed through bilateral ischioanal incisions. No pathology of the scrotum and groin was observed. The digital rectal examination provoked severe pain and was not diagnostic. The laboratory studies showed leukocytosis (white blood cells number, 18.000/ $\mu \mathrm{L}$; neutrophils, $97 \%$ ) and an increased level of C-reactive protein (CRP, $30 \mathrm{mg} / \mathrm{L}$ ). The computed tomography (CT) scan, which was performed 5 days prior to presentation, demonstrated a posterior horseshoe abscess and inflammation of the right pararectal space. Due to the patient's 
indolent clinical presentation, the complex perianal surgical wound and the persistent fever despite the multiple drainage procedures, a magnetic resonance imaging (MRI) of the lower abdomen and pelvis was performed. As a new finding, an abscess cavity was revealed in the right supralevator space (Fig. 1). Fluid collections and air were detected in the retropubic area and anterolateral extraperitoneal compartments which extended to the anterior abdominal wall (Fig. 2). Additionally, inflammation of the right internal obturator muscle was demonstrated as a clear-cut extension of the ischiorectal abscess (Fig. 3). No intraperitoneal pathology was identified. Regarding the perianal wound, the ischioanal cavities were sufficiently drained and a transphincteric fistula tract was identified in the posterior midline.

Initial resuscitation with crystalloids and intravenous broadspectrum antibiotics was followed by an examination under general anaesthesia. Prior to induction of general anesthesia, the patient received counsel regarding the emergency procedure, and written informed consent was obtained. Our institution's policy is that a case report does not meet the criteria of research that must be approved by the Health Sciences Institutional Review Board (IRB) therefore it is exempt from IRB review. In the operating room, thorough inspection and wash-out of the bilateral ischioanal spaces were possible through the previous incisions. Neither an additional surgical wound to the perineum nor a posterior midline sphincterotomy were performed. The anoscopic examination revealed the internal opening of a fistulous tract in the posterior midline (6 oclock in lithotomy position). A mallea-

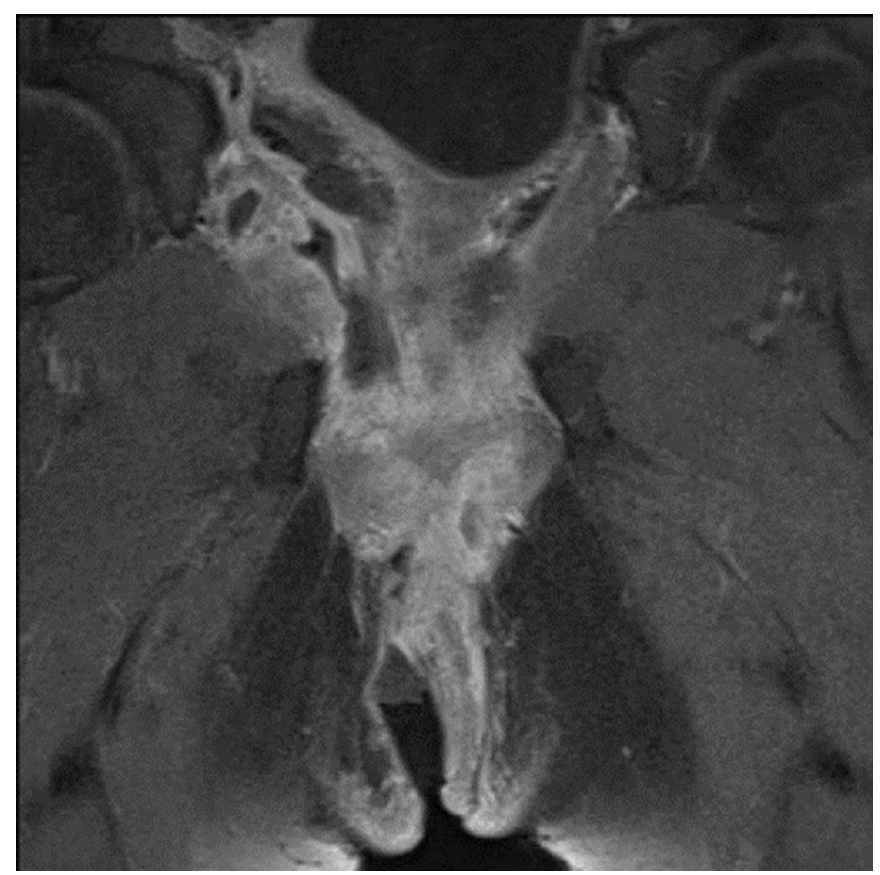

Fig. 1. Horseshoe abscess cavity involving the bilateral ischioanal spaces. ble probe was placed through the internal opening to the right deep postanal cavity, excluding any other potential limbs of the fistula. A vessel loop was placed as a loose seton drainage in the fistula tract (Fig. 4).

Because of the extraperitoneal fluid collections and air noted on the MRI report, a low midline incision was used to gain access to the extraperitoneal compartments. Intraperitoneal surgical manipulations were avoided. A fair amount of pus was drained from the anterolateral abdominal wall. Further exploration revealed a large right supralevator abscess in proximity to the internal obturator muscle. The pararectal and retropubic spaces were washed out and carefully dried off many times. A connection was created between the right retropubic space and the right ischiorectal fossa through the internal obturator muscle, providing adequate drainage of the interconnecting spaces. A careful dissection was performed at a distance from the obturator canal. A Foley catheter was placed through the anterolateral abdominal sidewall to the ischiorectal wound, allowing through and through irrigation and drainage (Fig. 5). The right levator ani muscle was not divided since the catheter was placed through the internal obturator muscle to the right ischiorectal space. Considering the septic condition of the patient and the extensive perianal wound, a diverting loop sigmoidostomy was created and sutured to the lateral abdominal sidewall. Multiple drains in the perineal wound as well as the Foley catheter inside the interconnecting spaces provided adequate drainage of the cavities during the postoperative period.

The patient remained hospitalized for eight days. The fever re-

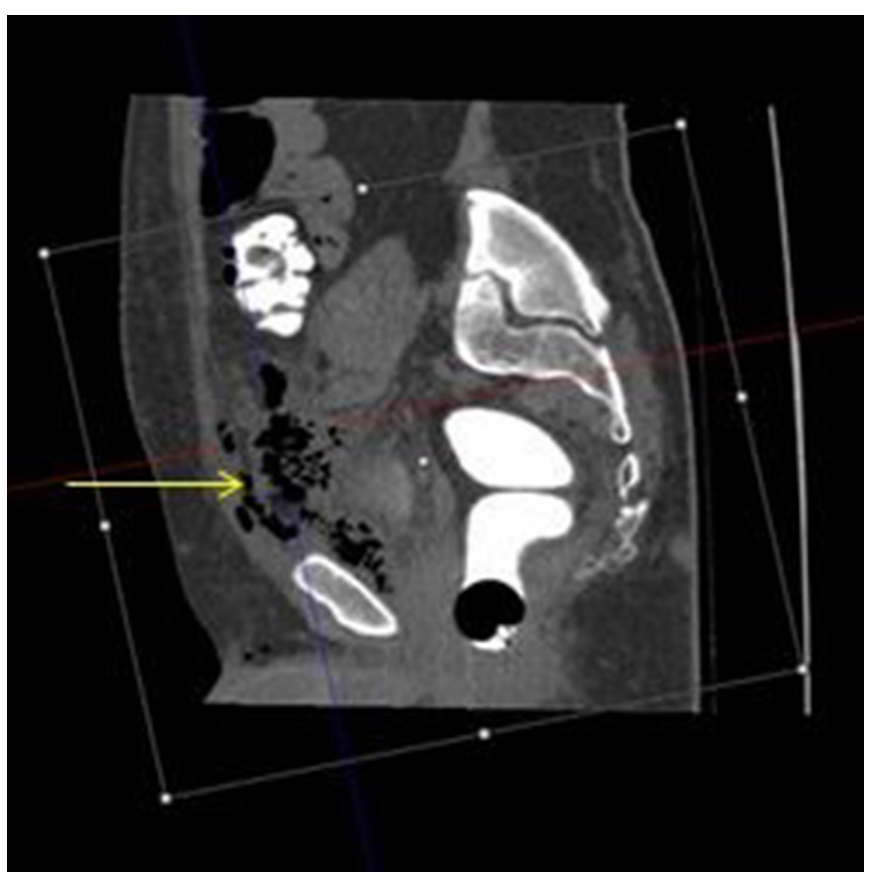

Fig. 2. Air and fluid (arrow) in the anterolateral extraperitoneal compartments. 


\section{A Rare Case of Posterior Horseshoe Abscess
Annals of \\ Coloproctology Christianna Oikonomou, et al.}

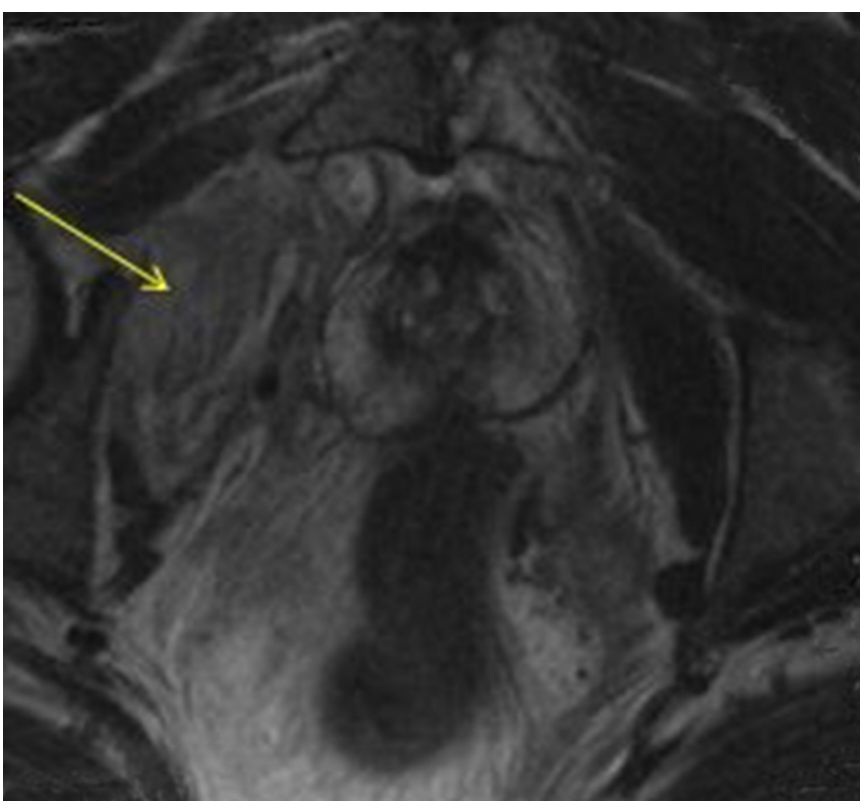

Fig. 3. Inflammation of the right internal obturator muscle (arrow).

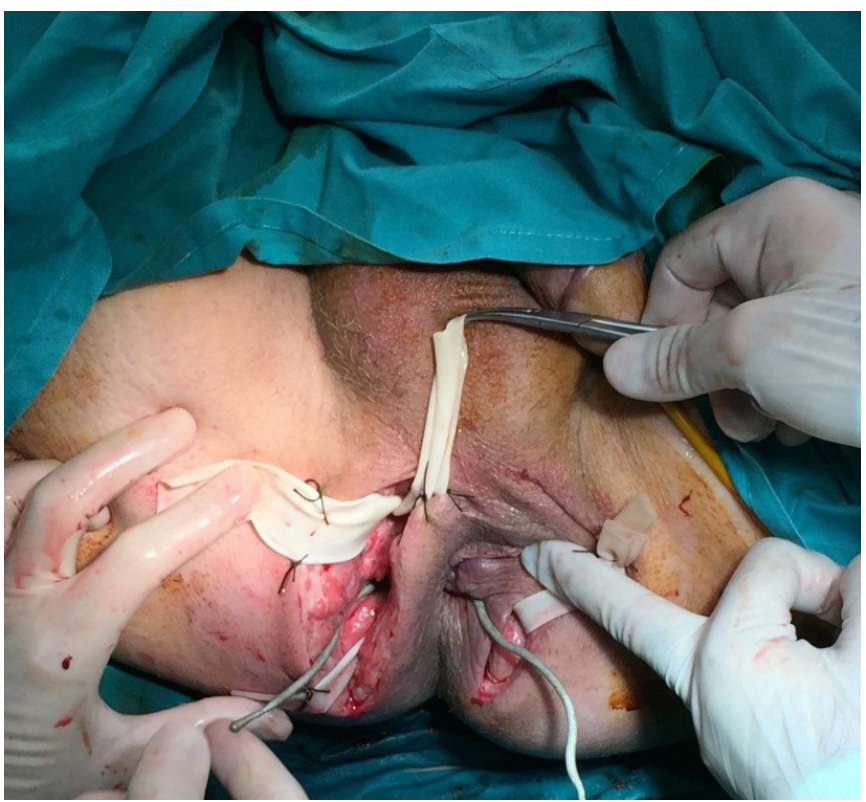

Fig. 4. Drainage of the ischioanal abscesses using drainages.

ceded on the second postoperative day. Serial irrigations through the Foley catheter and digital examinations separating the wound edges were performed. Considering the patient's progressive clini$\mathrm{cal}$ and laboratory improvement, no further surgical exploration was performed. The patient was discharged on the eighth postoperative day in good condition. He was encouraged to clean his wounds with tap water twice a day. The drains were removed five days later. One week after the drainage procedure, a CT scan con-

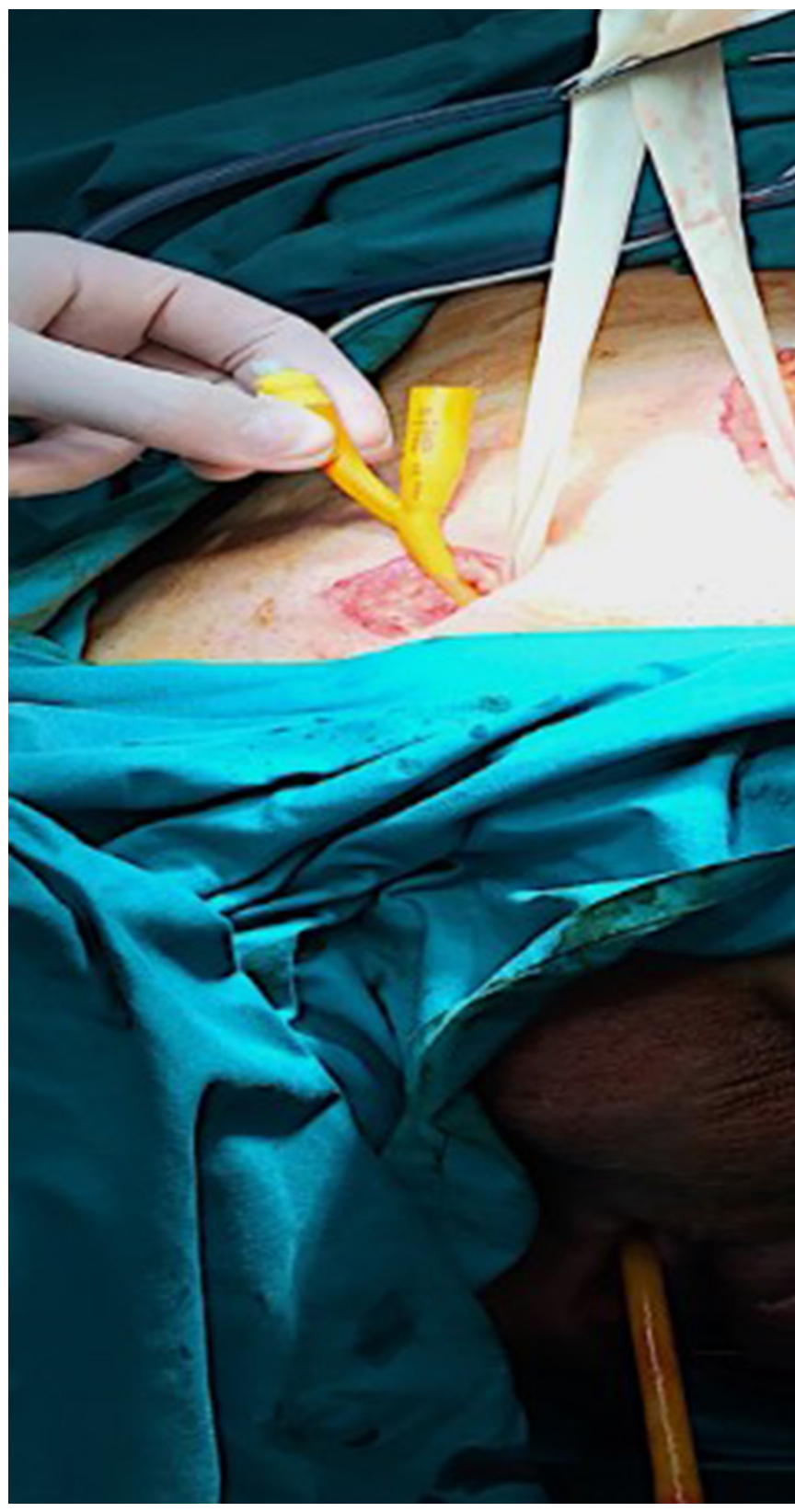

Fig. 5. A foley catheter passed from the right anterolateral extraperitoneal space to the ischioanal fossa through the internal obturator muscle.

firmed the radiologic amelioration. The inflammatory process was limited, and no abscess cavities were detected. The surgical wounds were kept open to heal by secondary intention and were found to be completely healed 8 weeks later. The seton drainage remained, tracking a transphincteric fistula-in-ano. A ligation of intersphincteric fistula tract (LIFT procedure) was performed. The patient was placed in the prone position. The vessel loop as a seton drainage was removed, and a metallic probe was inserted 
inside the tract. An incision was made at the intersphincteric groove. Identification and suture ligation of the tract were achieved. The wound was closed with nonabsorbable sutures. The postoperative course was uneventful. Four weeks later, closure of the loop sigmoidostomy was performed with no postoperative complications. No recurrence was observed during a follow-up of twenty months. Neither sphincteric dysfunction nor degree of incontinence was encountered.

\section{DISCUSSION}

High, complex perianal abscesses and fistulas have remained a challenging problem over the years, mainly due to the risk of postoperative incontinence and the high rates of recurrence.

A horseshoe abscess is a particularly aggressive type of perianal disease in which there is an erratic pattern of spread in a ring-like fashion to the deep postanal or even preanal space and then to the bilateral ischiorectal fossae by way of Colles fascia. This condition may represent $15 \%$ to $20 \%$ of fistulous lesions in the anorectal region [3]. Horseshoe abscesses have been associated with high rates of recurrence ranging between $18 \%-50 \%$. Inadequate drainage, failure to break up loculations, and/or failure to reveal multiple fistulous tracts increase the rate of persistence [4].

In the present case, the drainage of a posterior horseshoe abscess was complicated by a supralevator abscess and defusing purulent inflammation in the anterolateral extraperitoneal compartment. In our view, the previous serial drainage incisions of the horseshoe abscess triggered an upward spread of the infection via the relevant anatomical planes. Inappropriate preoperative planning resulted in inappropriate treatment and an extensive surgical wound. Spread of the infection can be predicted by anatomic routes and boundaries. In the literature, most authors concur that the infection follows an upward path from a septic perianal lesion through the intersphincteric plane and the diaphragmatic fascia of the levator ani muscle to the retrorectal space posteriorly or the superior pelvirectal space anteriorly [5].

Moreover, the supralevator space communicates anteriorly with the retropubic space (space of Retzius), laterally with the space of Bogros and posteriorly with the infrarenal retroperitoneal space [6]. The infection can directly spread through these interconnecting compartments.

The first step when managing a complex perianal abscess and fistulas is to define the anatomy and the extent of the purulent process. Clinical findings alone are insufficient, and the initial diagnosis as well as the differential diagnosis need adjunctive imaging modalities [4]. In the current report, the rarity of the case and the atypical presentation may have mimicked different disease entities, such as Fournier's gangrene or other necrotizing fasciitis. The latter infections travel along the Colles' fascia limited from the supralevator and retrorectal spaces. The MRI was highly accurate in detecting the extraperitoneal abscesses and useful in delineating the anatomical planes that offered highways for the in- fection to spread. The internal opening of the posterior fistula-inano was also identified. In our department, MRI is included in the preoperative assessment for patients with complex benign colorectal diseases, especially when high fistulous tracts are encountered. In the literature, endoanal ultrasound is an alternative modality used to determine the fistulous tracts and perirectal pathology, especially when the diagnosis is still questionable following the clinical examination and anoscopy. CT scan remains a valuable tool, especially when intraperitoneal involvement is suspected [4].

According to the relevant literature, various procedures have been proposed for the surgical management of a horseshoe fistulous abscess. Historically, in 1965, Hanley [7] proposed a primary fistulotomy and counter drainage as the procedure of choice. This procedure was correlated with high rates of recurrence and incontinence. Later, in 1984, Hanley modified the procedure. He suggested, as a first step, the use of a seton drainage to intercept the spread of suppuration to be followed, as a second step, by an outpatient tightening of the seton to sever the sphincter muscle, stimulating a fibrous reaction. The risk of incontinence as well as the persistence rates was lower in patients undergoing the modified procedure [8].

In the present case, bilateral drainage of the horseshoe abscess was followed by placement of a seton drainage in the posterior fistula-in-ano in order to eradicate anorectal sepsis. As a second procedure, a sphincter sparing approach was chosen. LIFT is a novel technique that aims to maintain the anal sphincter intact, and the success rates range from $63 \%-94.4 \%[9,10]$. Tan et al. [11] reported a $70.6 \%$ success rate when managing deep postanal sepsis via a primary or secondary intersphincteric approach.

Regarding the surgical management of supralevator and extraperitoneal abscesses, the reports are sparse. In 1943, Gaston and Lyman [12] supported that drainage of the anterior-pelvirectal and posterior-retrorectal space should be performed by making incisions lateral to the anal canal, in parallel and slightly lateral to the coccyx respectively. Moreover, Gaston and Lyman [12] reported that the fat of the ischiorectal fossa should be traversed with sharp and blunt dissection, after which transverse division of the levator ani muscle should permit access to the supralevator space. In a series of 506 patients with anorectal suppurations, Prasad et al. [2] presented 46 patients treated for supralevator abscess. An elliptical incision of the perianal skin was followed by exploration and adequate drainage of the ischiorectal fossa, with occasional placement of multiple drains. Access to the supralevator space was gained after excising a portion of the levator ani. To avoid creating an iatrogenic supralevator fistula, drainage of the supralevator abscesses through synchronous incisions to the rectum and ischiorectal fossa was not performed. Following this procedure, recurrence rate was very low [2]. In a more recent report, successful evacuation of preperitoneal pus in the space of Retzius was achieved through lower abdominal incision and exploration of the relevant extraperitoneal spaces [6]. 
Lastly, in most reports, the creation of a diverting stoma is a solution mainly performed when Crohn disease is encountered [13]. Marks et al. [14] analyzed 11 deaths attributed to fistulous abscess, and he reported that feces diversion did not prevent the septic complications. In the current case, the extensive perianal surgical wound and the complexity of the suppuration process were major determinants in the decision to perform a loop sigmoidostomy in order to prevent fecal soiling into the wound. The patient's recovery was uneventful; further surgical procedures were not necessary, and the infection was resolved. The closure of the colostomy was performed without any complication.

In conclusion, management of high complex perianal abscess and fistula demand vigorous initial supportive therapy and a surgeon with a deep knowledge of the relevant anatomy. Awareness of the diagnosis and the possible routes of spread as well as expertise in the surgical field are fundamental elements for a successful outcome. Preoperative planning must be directed by radiological studies to avoid multiple inappropriate procedures, high recurrence rates and extensive surgical wounds to the perineum and the anal sphincters.

\section{CONFLICT OF INTEREST}

No potential conflict of interest relevant to this article was reported.

\section{REFERENCES}

1. Parks AG, Gordon PH, Hardcastle JD. A classification of fistulain-ano. Br J Surg 1976;63:1-12.

2. Prasad ML, Read DR, Abcarian H. Supralevator abscess: diagnosis and treatment. Dis Colon Rectum 1981;24:456-61.

3. Umoh NJ. Surgical management of deep postanal abscess and horseshoe fistula of cryptoglandular origin - a review. Clin Surg 2017;2:1570.
4. Steele SR, Kumar R, Feingold DL, Rafferty JL, Buie WD; Standards Practice Task Force of the American Society of Colon and Rectal Surgeons. Practice parameters for the management of perianal abscess and fistula-in-ano. Dis Colon Rectum 2011;54: 1465-74.

5. Mirilas P, Skandalakis JE. Surgical anatomy of the retroperitoneal spaces part II: the architecture of the retroperitoneal space. Am Surg 2010;7633-42.

6. Mentzer CJ, Yon JR, King R, Warren JA. Complex perirectal abscess extending to the preperitoneum and space of retzius. GHS Proc 2016;1:49-51.

7. Hanley PH. Conservative surgical correction of horseshoe abscess and fistula. Dis Colon Rectum 1965;8:364-8.

8. Browder LK, Sweet S, Kaiser AM. Modified Hanley procedure for management of complex horseshoe fistulae. Tech Coloproctol 2009;13:301-6.

9. Rohit DK, Jain S, Pandey G. Effectiveness of ligation of intersphincteric fistula tract (LIFT) in the management of fistulas in ano. Int Surg J 2017;4:3951-5.

10. Rojanasakul A, Pattanaarun J, Sahakitrungruang C, Tantiphlachiva K. Total anal sphincter saving technique for fistula-in-ano; the ligation of intersphincteric fistula tract. J Med Assoc Thai 2007; 90:581-6.

11. Tan KK, Koh DC, Tsang CB. Managing deep postanal space sepsis via an intersphincteric approach: our early experience. Ann Coloproctol 2013;29:55-9.

12. Gaston E, Lyman W. Supralevator abscess. New Engl Med 1943; 229:613-9.

13. Rosen SA, Colquhoun P, Efron J, Vernava AM 3rd, Nogueras JJ, Wexner SD, et al. Horseshoe abscesses and fistulas: how are we doing? Surg Innov 2006;13:17-21.

14. Marks G, Chase WV, Mervine TB. The fatal potential of fistulain-ano with abscess: analysis of 11 deaths. Dis Colon Rectum 1973;16:224-30. 